\title{
The nature of $\Lambda(1405)$ hyperon resonance in chiral dynamics
}

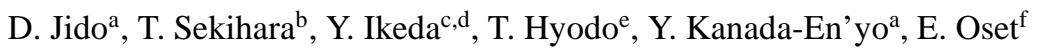 \\ ${ }^{a}$ Yukawa Institute for Theoretical Physics, Kyoto University, Kyoto 606-8502, Japan \\ ${ }^{b}$ Department of Physics, Graduate School of Science, Kyoto University, Kyoto, 606-8502, Japan \\ ${ }^{c}$ Depertment of Physics, University of Tokyo, Tokyo 113-0033, Japan \\ ${ }^{d}$ Nishina Center for Accelerator-Based Science, Institute for Physical and Cemical Research (RIKEN), Wako, Saitama \\ 351-0198, Japan \\ ${ }^{e}$ Department of Physics, Tokyo Institute of Technology, Meguro 152-8551, Japan \\ ${ }^{f}$ Departamento de Física Teórica and IFIC, Centro Mixto Universidad de Valencia-CSIC, Institutos de Investigación de \\ Paterna, Aptdo. 22085, 46071 Valencia, Spain
}

\begin{abstract}
The nature of the $\Lambda(1405)$ is discussed based on the unitarised coupled-channels approach with chiral dynamics (chiral unitary model). This approach describes the $\bar{K} N$ scattering cross sections and the $\Lambda(1405)$ spectra phenomenologically very well. With this successful description of $\Lambda(1405)$, it is found that the $\Lambda(1405)$ is composed by two resonance states having different coupling nature to the meson-baryon states. As a consequence, the resonance position in the $\pi \Sigma$ invariant mass spectrum depends on the initial channel of the $\Lambda(1405)$ production. To observe the $\Lambda(1405)$ initiated by the $\bar{K} N$ channel, $K^{-} d \rightarrow \Lambda(1405) n$ is one of the most favorable reactions. Hadronic molecule states with kaons are also discussed by emphasizing an important role of $\Lambda(1405)$ as a quasibound state of $\bar{K} N$
\end{abstract}

Keywords: Structure of $\Lambda(1405)$, Kaon induced $\Lambda(1405)$ production, Chiral unitary model, $K \bar{K} N$ quasibound state

PACS: $14.20 . \mathrm{Jn}, 25.80 . \mathrm{Nv}, 13.75 . \mathrm{Jz}, 12.39 . \mathrm{Fe}, 13.30 . \mathrm{Eg}$

\section{Introduction}

The nature of the $\Lambda(1405)$ resonance is an important issue particularly to understand $\bar{K}$ nucleus interactions. Being located just below the threshold of $\bar{K} N$, the $\Lambda(1405)$ is a key resonance for the study of subthreshold kaons in nuclei. Due to the decay of the $\Lambda(1405)$ to $\pi \Sigma$, for the structure of the $\Lambda(1405)$, both $\pi \Sigma$ and $\bar{K} N$ dynamics are essential. The $\Lambda(1405)$ has been a historical example of a dynamically generated resonance in meson-baryon coupled-channels dynamics with $S=-1$ [1]. Modern treatments based on chiral dynamics with a unitary framework, reproduce well the observed spectrum of the $\Lambda(1405)$ together with cross sections of $K^{-} p$ to various channels $[2,3,4,5,6,6,8,9]$. This method is based on the chiral perturbation theory of 
an effective theory of QCD at low energies giving fundamental interaction of meson and baryon, and scattering theory, such as the $N / D$ method, to guarantee unitarity on the scattering amplitudes. Analyticity of the scattering amplitude is also essentially important to obtain subthreshold amplitudes and to investigate resonance properties in the complex energy plane. The obtained amplitude reproduces the meson-baryon scattering well and provides $\Lambda(1405)$ as a dynamically generated resonance in $s$-wave meson-baryon scattering.

On the success of the theoretical description of the $\Lambda(1405)$, it it worth noting the following facts; 1$)$ the available experimental data to constrain theoretical descriptions of the scattering amplitude with $S=-1$ around the $\Lambda(1405)$ energies are the $\bar{K} N$ scattering and kaonic hydrogen data. Thus, the present theoretical description lacks experimental information of $\pi \Sigma \rightarrow \pi \Sigma$, which may be important to study $\bar{K}$ in few-body nuclear systems. 2) the famous $\Lambda(1405)$ spectrum given in Ref. [10] is a $\pi^{-} \Sigma^{+}$invariant mass observed in the $\Sigma^{+}(1660) \rightarrow \Lambda(1405) \pi^{+}$decay. Therefore, the initial channel to produce $\Lambda(1405)$ is not clear. As seen below, since the $\Lambda(1405)$ spectrum shape depends on the production channel, it is extremely important to control the initial channel experimentally and to investigate the production mechanism theoretically for the good understanding of the nature of $\Lambda(1405)$.

\section{The structure of $\Lambda(\mathbf{1 4 0 5})$}

In this section, we explain the findings about the nature of $\Lambda(1405)$ obtained by the chiral unitary approach.

\subsection{Double pole nature of $\Lambda(1405)$}

Resonances are expressed as poles of the scattering amplitude in the complex energy plane. In the chiral unitary approach for the meson-baryon system developed by Ref. [4, 5], the scattering amplitude is obtained in an analytic form, so that one can easily perform analytic continuation of the scattering amplitude to the complex energy plane. In Ref. [7] it was found that the $\Lambda(1405)$ seen in the $\pi \Sigma$ invariant mass spectra consists of two resonance poles located at the $\Lambda(1405)$ energies, and these two state have different coupling properties to the meson-baryon states; one is located at $1390-66 i \mathrm{MeV}$ (pole 1) with a larger width having strong coupling to the $\pi \Sigma$ state, ant the other pole is at higher energy $1426-16 i$ (pole 2) MeV with a narrower width and dominantly couples to the $\bar{K} N$ state. Because these two states closely appear with the large widths, one cannot observe these states separately in the $\pi \Sigma$ invariant mass spectra (See Fig. 1 b)). What we can see is a single peak structure provided by the interference of these two states, that is the $\Lambda(1405)$ spectrum observed in experiments.

The reason that there exist two poles around the $\Lambda(1405)$ energies is that there are two attractive channels with $S=-1$ and $I=0$ in $s$-wave at these energies [7], $\bar{K} N$ and $\pi \Sigma$. These two attractive channels provide the two resonance states. It is also found in a recent work [11] that the $\Lambda(1405)$ can be described essentially by two coupled channels of $\bar{K} N$ and $\pi \Sigma$ rather than a complete $\mathrm{SU}(3)$ configuration by four channels, $\bar{K} N, \pi \Sigma, \eta \Lambda$ and $K \Xi$ with $I=0$. In addition, without the channel couplings between $\bar{K} N$ and $\pi \Sigma$, one has a bound state in the $\bar{K} N$ channel below the $\bar{K} N$ threshold and a resonance in the $\pi \Sigma$ channel above the $\pi \Sigma$ threshold. These two states appear very close to the original states obtained in coupled channels. Therefore, the essential ingredients of $\Lambda(1405)$ is the $\bar{K} N$ bound state and $\pi \Sigma$ resonant correlation. This is the reason for the double pole structure of $\Lambda(1405)$ and the different coupling nature of the two states. 
(a) Comparison of two spectra

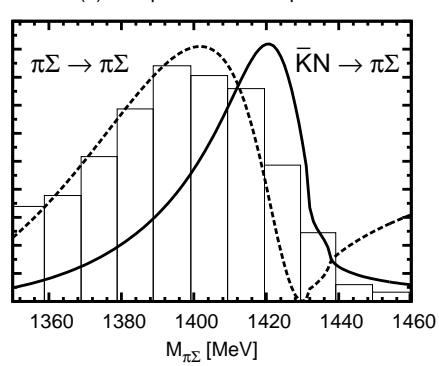

(b): $\pi \Sigma \rightarrow \pi \Sigma$

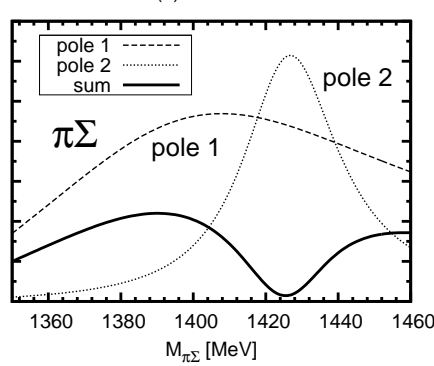

(c): $\mathrm{K}^{\mathrm{bar}} \mathrm{N} \rightarrow \pi \Sigma$

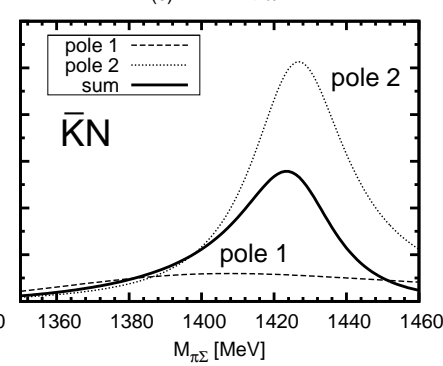

Figure 1: $\pi \Sigma$ invariant mass spectra with $I=0$ in arbitrary units [7]. (a): chiral unitary model calculation of the $\pi \Sigma$ invariant mass spectra of $\bar{K} N \rightarrow \pi \Sigma$ (solid line) and $\pi \Sigma \rightarrow \pi \Sigma$ (dashed line). The histogram denotes an experimental data given in Ref. [10]. (b) and (c): simple model calculations of the $\pi \Sigma$ invariant mass spectra initiated by $\pi \Sigma$ and $\bar{K} N$, respectively, in which the scattering amplitudes are given by two Breit-Wigner terms. The dashed (dotted) line denotes the spectrum only with pole 1 (pole 2) term, while the solid line shows the spectrum calculated by coherent sum of pole 1 and 2. The Breit-Wigner parameters are determined by the chiral unitary model. For the details, see Ref. [7].

\subsection{Channel dependence of $\Lambda(1405)$ spectrum}

The presence of the two poles having different coupling properties results in initial channel dependence of the $\Lambda(1405)$ peak position in the $\pi \Sigma$ invariant spectrum. In Fig. 1 a), we show the $\pi \Sigma$ invariant mass spectra with $I=0$ initiated by two different channels, $\bar{K} N$ and $\pi \Sigma[7]$. The figure shows that the $\Lambda(1405)$ peak is seen in the different energies; For the $\pi \Sigma$ initiated spectrum, the resonance peak appears at around $1405 \mathrm{MeV}$, whereas in the $\bar{K} N \rightarrow \pi \Sigma$ channel, the peak position is located higher at around $1420 \mathrm{MeV}$ instead of $1405 \mathrm{MeV}$. This is because, for the $\pi \Sigma$ channel, both of the lower and higher poles contribute to the spectrum, while the $\bar{K} N$ system selectively couples to the higher pole, as seen in Figs. 1(b) and 1(c), where we show decomposition of the $\Lambda(1405)$ spectrum into each pole contribution. The decomposition is performed by the model calculation in which the amplitudes are given by Breit-Wigner terms for pole 1 and 2 together with the resonance parameters, such as mass, width and couplings, determined by the chiral unitary model [7]. In this way, the resonance position in the $\pi \Sigma$ invariant mass depends on the channels to produce the $\Lambda(1405)$. Particularly, the finding that the $\Lambda(1405)$ resonance appears at $1420 \mathrm{MeV}$ in the $\bar{K} N$ initiated channel is important for kaon-nucleus systems, since the resonance appearing in the $\bar{K} N$ channel is the one relevant for the kaon-nucleon interaction.

One of the experimental confirmation of the double pole nature of the $\Lambda(1405)$ is to compare the $\Lambda(1405)$ spectra in different channels. Especially, as we already discussed above, one of the important consequences is that the resonance in the spectrum initiated by the $\bar{K} N$ channel appears at $1420 \mathrm{MeV}$. Therefore, it is interesting to see the resonance position in the $\Lambda(1405)$ production initiated by $\bar{K} N$. However, direct production $\bar{K} N \rightarrow \Lambda(1405)$ is kinematically forbidden, since the $\Lambda(1405)$ resonance appears below the $\bar{K} N$ threshold. This fact leads us to indirect productions of $\Lambda(1405)$. But, in the indirect production, it is necessary to investigate the $\Lambda(1405)$ production mechanism theoretically to recognize the initial channels of the $\Lambda(1405)$ production.

\section{3. $\Lambda(1405)$ observed in $\bar{K} N$ channel}

Here we discuss the $K^{-}$induced production of $\Lambda(1405)$ with a deuteron target, $K^{-} d \rightarrow$ $\Lambda(1405) n$, proposed in Ref. [12], to see the $\Lambda(1405)$ in the $\bar{K} N$ channel. In this reaction, the final neutron takes energy out from the initial kaon. The produced $\Lambda(1405)$ decays into $\pi \Sigma$ 

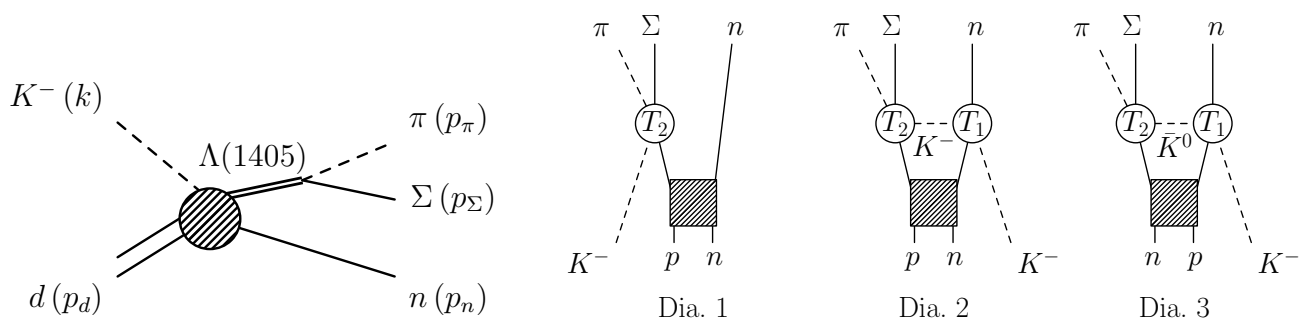

Dia. 2

Dia. 3

Figure 2: Diagrams for the calculation of the $K^{-} d \rightarrow \pi \Sigma n$ reaction. The right three are the Feynman diagrams for our calculation. $T_{1}$ and $T_{2}$ denote the scattering amplitudes for $\bar{K} N \rightarrow \bar{K} N$ and $\bar{K} N \rightarrow \pi \Sigma$, respectively.

with $I=0$ as shown in Fig. 2, and the $\Lambda(1405)$ is identified by the $\pi \Sigma$ invariant mass spectra. The relevant contribution for the $\Lambda(1405)$ production in this reaction is given by three diagrams shown in Fig. 2. The amplitude $T_{1}$ and $T_{2}$ denote $s$-wave scattering amplitudes of $\bar{K} N \rightarrow \bar{K} N$ and $\bar{K} N \rightarrow \pi \Sigma$, respectively, and the $\Lambda(1405)$ resonance is involved in the amplitude $T_{2}$. Thus, it is important noting that, in this reaction, the $\Lambda(1405)$ is produced selectively by the $\bar{K} N$ channel. This is because the strangeness is brought into the system by the initial kaon.

Diagram 1 of Fig. 2 expresses the $\Lambda(1405)$ production in the impulse approximation. Diagrams 2 and 3 are for two-step processes with $\bar{K}$ exchange. For the energetic incident $K^{-}$with several hundreds $\mathrm{MeV} / \mathrm{c}$ momentum in the lab. frame, the contribution of diagram 1 (direct production) is expected to be very small, since the $\Lambda(1405)$ is produced by a far off-shell nucleon and the deuteron wavefunction has tiny component of such a nucleon. In contrast to the direct production, in the double scattering diagrams, the large energy of the incident $K^{-}$is carried away by the final neutron and the exchanged kaon can have a suitable energy to create the $\Lambda(1405)$ colliding with the other nucleon in the deuteron. Double scattering diagrams with pion exchanges hardly contribute to the $\Lambda(1405)$ production, since $\Sigma$ and $\pi$ are emitted separately from the $T_{1}$ and $T_{2}$ amplitudes. Such diagrams may give smooth backgrounds in the $\pi \Sigma$ invariant mass spectra.

We show, in Fig. 3, the $\pi^{+} \Sigma^{-}$invariant-mass spectrum in arbitrary units at $800 \mathrm{MeV} / \mathrm{c}$ incident $K^{-}$momentum and compare our theoretical calculation with the experimental data. The data are taken from the bubble chamber experiment at $K^{-}$momenta between 686 and $844 \mathrm{MeV} / \mathrm{c}$ [13]. In the analysis of the experiment, the resonance contribution was determined by fitting a relativistic Breit-Wigner distributions and a smooth background parametrized as a sum of Legendre polynomials to the data. We show, in Fig. 3, the resonance (foreground) contributions for the experimental data which are obtained by subtracting the background contributions from the actual data points given in the paper. The solid line denotes our theoretical calculation with the scattering amplitudes $T_{1}$ and $T_{2}$ obtained by chiral unitary approach. The spectrum shape obtained in this calculation agrees with that of the experimental observation. Especially it is very interesting to see that the peak position, which comes from the $\Lambda(1405)$ production, appears around $M_{\pi \Sigma}=1420 \mathrm{MeV}$ instead of $1405 \mathrm{MeV}$. This is one of the strongest evidences that the resonance position of the $\Lambda(1405)$ depends on the initial channel of meson and baryon, and supports the double pole nature of the $\Lambda(1405)$, in which the higher state sitting in $1420 \mathrm{MeV}$ strongly couples to the $\bar{K} N$ channel.

The bump structure seen around $M_{\pi \Sigma}=1400 \mathrm{MeV}$ in data is related to the $p$-wave contributions coming from the $\Sigma^{*}(1385)$ production [12, 14], which were not taken into account in the present calculation. In Ref. [12], an estimation of a possible influence of the $\Sigma(1385)$ resonance on the $\Lambda(1405)$ spectrum appearing around $1420 \mathrm{MeV}$ was discussed by calculating the $\Sigma(1385)$ 


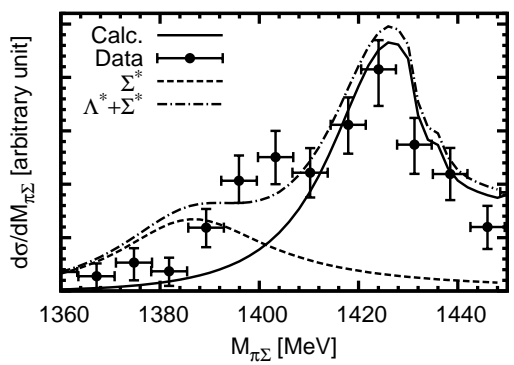

Figure 3: $\pi \Sigma$ invariant mass spectra of $K^{-} d \rightarrow \pi^{+} \Sigma^{-} n$ in arbitrary units at $800 \mathrm{MeV} / \mathrm{c}$ incident $K^{-}$momentum. The solid line denotes the present calculation. The data are taken from the bubble chamber experiment at $K^{-}$momenta between 686 and $844 \mathrm{MeV} / \mathrm{c}$ given in Ref. [13]. A possible inference of the $\Sigma(1385)$ resonance to the $\Lambda(1405)$ spectrum of $K^{-} d \rightarrow \pi^{+} \Sigma^{-} n$ at $800 \mathrm{MeV} / \mathrm{c}$ incident $K^{-}$momentum. The $\Sigma(1385)$ spectrum (dashed line) is calculated by the Breit-Wigner amplitude. The dash-dotted lines denotes an incoherent sum of the $\Lambda(1405)$ and $\Sigma(1385)$ spectrum.

spectrum in the Breit-Wigner formulation with the mass $1385 \mathrm{MeV}$ and the width $37 \mathrm{MeV}$ and the phase space factor $\left|\vec{p}_{\pi}^{*}\right|\left|\vec{p}_{n}\right|$. Summing up the spectra of the $\Lambda(1405)$ and $\Sigma(1385)$ incoherently, they find that the peak structure at $1420 \mathrm{MeV}$ is not affected by the $\Sigma(1385)$ contribution as seen in Fig. 3 (dash-dotted line). In this estimation, they have adjusted the height of the $\Sigma(1385)$ spectrum so as to reproduce the observed bump structure around $1390 \mathrm{MeV}$. A further quantitative calculation of the $\Sigma(1385)$ spectrum by taking into account of the $\Sigma(1385)$ production mechanism in the present reaction with the $p$-wave contributions following Ref. [15], will be coming soon [14].

\subsection{Model dependence of pole positions and significance of $\pi \Sigma$ scattering data}

The higher pole located at around $1420 \mathrm{MeV}$ is essentially a $\bar{K} N$ quasibound state and strongly couples to $\bar{K} N$. It has been pointed out in Ref. [11] that the position of the higher pole has less model dependence among different calculations based on coupled-channels approach with chiral dynamics, while these calculations predict different positions for the lower pole which couples to $\pi \Sigma$ strongly (see Fig. 8 in Ref. [11]). This is because, in these model calculations, $K^{-} p$ scattering data are used to fix the model parameters and the properties of the $\bar{K} N$ interaction are well constrained by exciting data. It was also pointed out in a different approach [16] that a pole appears at around $1420 \mathrm{MeV}$ in the $\bar{K} N$ scattering amplitude which reproduces the $\bar{K} N$ scattering lengths. Contrarily, we have few data available for $\pi \Sigma \rightarrow \pi \Sigma$ scattering so far.

The lack of the $\pi \Sigma$ scattering data makes the properties of the lower pole less controlled in theoretical calculations. Any information of the $\pi \Sigma$ scattering is desired to fix further the nature of the $\Lambda(1405)$. For instance, the threshold properties of the $\pi \Sigma$ scattering, such as the scattering length and effective range, could give suitable constraint [17]. In Table 1, we show values of the $\pi \Sigma$ scattering length with $I=0$ calculated using various chiral coupled-channels models. The values of the scattering length are substantially dependent on the models, which predict also different pole positions of the lower pole.

A more interesting question is whether the pole in the $\pi \Sigma$ is really a resonance or not. The chiral unitary approach predicts that the $\pi \Sigma$ strong correlation forms a resonance state found above the $\pi \Sigma$ threshold. Yet we do not have the experimental confirmation of the $\pi \Sigma$ resonance pole. If the $\pi \Sigma$ interaction were unexpectedly strong enough, there could exist a $\pi \Sigma$ virtual state below 


\begin{tabular}{|c|cccc|c|}
\hline model & BNW [8] & ORB [5] & HNJH [6] & BMN [9] & virtual state \\
\hline$a_{\pi \Sigma}[\mathrm{fm}]$ & 0.517 & 0.789 & 0.692 & 0.770 & $\sim 5$ \\
$z_{1}[\mathrm{MeV}]$ & $1388-39 i$ & $1389-64 i$ & $1400-76 i$ & $1440-76 i$ & $1325(\mathrm{~V})$ \\
\hline
\end{tabular}

Table 1: Model calculations of the $I=0 \pi \Sigma$ scattering lengths $a_{\pi \Sigma}$ using various chiral coupled-channels models. (For the detail, see Ref. [17]). $z_{1}$ denotes the pole position of the lower resonance. The right row shows the value of the $\pi \Sigma$ scattering length calculated under the assumption that there is a virtual state around $10 \mathrm{MeV}$ below the $\pi \Sigma$ threshold.

the $\pi \Sigma$ threshold in stead of the resonance state. In this case, the $\Lambda(1405)$ would be consisted of one single pole. Alternatively, for the $s$-wave interaction, energy-independent potentials can provide only virtual states. Thus, in case that the energy-dependence of the $\pi \Sigma$ interaction would be weak in the $\pi \Sigma$ threshold energies, the $\pi \Sigma$ attraction could provide a virtual state. This sort of meson-baryon amplitude is studied in Ref. [16]. The question of either resonance or virtual state in the $\pi \Sigma$ correlation can be answered by the magnitude of the $\pi \Sigma$ scattering length. If the virtual state exists close to the threshold, the scattering length is in order of $5 \mathrm{fm}$, while, as we already saw, for the resonance case, the scattering length is around $0.5 \mathrm{fm}$, which is one order of magnitude small.

Understanding the $\pi \Sigma \rightarrow \pi \Sigma$ scattering properties is important not only for the $\Lambda(1405)$ structure but also for $\bar{K}$ in few-body systems, such as $\bar{K} N N[18,19,16,20]$. As discussed in Ref. [21] for the $K \bar{K} N$ system, if $\bar{K}$ few-body states are weakly bound, the $\bar{K}$ and nucleons are the essential constituents, and $\pi$ and $\Sigma$ can be regarded as nonactive degrees of freedom. Thus, the coupled channels effect of $\pi \Sigma$ can be incorporated into the effective $\bar{K} N$ interaction as done in Ref. [11], and the few-body bound state is essentially obtained in the single channel problem. However, since the $\pi \Sigma$ threshold is located $100 \mathrm{MeV}$ below the threshold of the $\bar{K} N$, if the $\bar{K}$ few-body systems turn out to be bound with a large binding energy, inclusion of the $\pi \Sigma$ coupled channel effect to theoretical calculations are unavoidable [22], and there in-vacuum $\pi \Sigma \rightarrow \pi \Sigma$ scattering data are necessary as a fundamental input.

\section{5. $\Lambda(1405)$ as meson-baryon quasibound state}

The third consequence of the chiral unitary model is that $\Lambda(1405)$ is almost purely a quasibound state of the meson and baryon, and quark components are much less important. A recent analysis [23] have shown that, although in the chiral unitary approach only the dynamics of meson and baryon is considered, the formulation implicitly involves some other components than the meson and baryon considered in the model space of the framework. The work [23] also discussed how to exclude the implicit components in theoretical description of scattering amplitudes, and compared the pole positions of the $\Lambda(1405)$ in the theoretical amplitude with those of the scattering amplitude obtained phenomenologically so as to reproduce the observed $\bar{K} N$ scattering. They find that these two descriptions provide very similar pole positions of $\Lambda(1405)$ and conclude that the $\Lambda(1405)$ is predominantly described by the meson-baryon component.

Owing to the meson-baryon quasibound structure, the $\Lambda(1405)$ has a different nature than typical baryon resonances which may have largely three-quark components. For instance, as a theoretical analysis, the behavior with the number of colors $\left(N_{c}\right)$ of the $\Lambda(1405)$ has been discussed in Ref. [24], and it has been found that the $N_{c}$ behavior of the decay widths is different from the general counting rule for a $q q q$ state. Another example is the size of the $\Lambda(1405)$. In Ref. [25], the electromagnetic mean squared radii of the $\Lambda(1405)$ were calculated. They found that the electric radius of the $\Lambda(1405)$ is about three times larger than that of the neutron. This is 
a consequence of the small binding energy $(10 \sim 30 \mathrm{MeV})$, in which the constituent kaon in the $\Lambda(1405)$ is an almost real particle surrounding around the nucleon.

\section{3. $\Lambda(1405)$ in few-body systems}

The $\Lambda(1405)$ can be an building-block of $\bar{K}$ nuclear few-body systems, since one of the pole in the $\Lambda(1405)$ is a quasibound state of $\bar{K} N$. It is quite interesting that the $\Lambda(1405)$ can be reproduced by nonrelativisitic potential models for the $\bar{K} N$ system [18, 11]. This is because the binding energy $(10 \sim 30 \mathrm{MeV})$ is not so large in comparison with typical hadron energy scale. (In other words, the kaon kinetic energy in the $\bar{K} N$ bound system is much smaller than the kaon mass). Thus, if the few-body systems also have not so large binding energies, the single channel potential model is one of the reasonable approached for the kaonic few-body systems, as done in Refs. [18, 20] for $\bar{K} N N$. This picture will be broken down if the binding energy is so large that the other channels become active. In such a case, the coupled channels effects should be properly treated in theoretical calculations, as done in Refs. [19, 16] for $\bar{K} N N$.

Here we would like to present other kaonic few-body systems, $K \bar{K} N$ and $\bar{K} \bar{K} N$ systems with $I=1 / 2$ and $J^{P}=1 / 2^{+}$. In Ref. [21], these systems were investigated in a nonrelativistic threebody potential model under the assumption that the $\Lambda(1405)$ resonance and the scalar mesons, $f_{0}(980), a_{0}(980)$, are reproduced as quasibound states of $\bar{K} N$ and $K \bar{K}$, respectively. The effective two-body interactions are described by complex-valued functions representing the open channels, $(\pi \Lambda, \pi \Sigma)$ for $\bar{K} N$ and $(\pi \pi, \pi \eta)$ for $K \bar{K}$. They found a quasibound state of the $K \bar{K} N$ system around $1910 \mathrm{MeV}$ below all of the meson-baryon decay threshold energies of the $\Lambda(1405)+K$, $f_{0}(980)+N$ and $a_{0}(980)+N$ states, which means that the obtained bound state is stable against breaking up to the subsystems. This quasibound state was also confirmed later by a more sophisticated calculation using a relativistic Faddeev approach [26]. For the $\bar{K} \bar{K} N$ system, the binding energy from the $\Lambda(1405)+\bar{K}$ threshold was found to be as small as a few $\mathrm{MeV}$ due to the strong repulsion $\bar{K} \bar{K}$ with $I=1[21]$.

For the structure of the $K \bar{K} N$ system, it was found that the $\bar{K} N$ and $K \bar{K}$ subsystems are dominated by $I=0$ and $I=1$, respectively, and that these subsystems have very similar properties with those in the isolated two-particle systems. This leads to the picture that the $K \bar{K} N$ system can be interpreted as coexistence state of $\Lambda(1405)$ and $a_{0}(980)$ clusters, and $\bar{K}$ is a constituent of both $\Lambda(1405)$ and $a_{0}(980)$ at the same time, as seen in the $\bar{K} N N$ sysytem [27, 20]. Consequently, the binding energy and width of the $K \bar{K} N$ state is almost the sum of those in $\Lambda(1405)$ and $a_{0}(980)$. It is also found that the inter-hadron distances in the $K \bar{K} N$ state are larger than $2 \mathrm{fm}$, which is comparable to typical nucleon-nucleon distances in nuclei. Therefore, the $K \bar{K} N$ system more spatially extends than typical baryons described by quark models. These features are caused by weak binding of the three hadrons, for which the $K N$ repulsion plays an essential role.

The finding that the $\Lambda(1405)$ keeps its properties in few-body systems motivates that picture that the $\Lambda(1405)$ resonance can be a doorway state of $\bar{K}$ absorption to nuclear systems [28]. Especially the coupling strengths of the $\Lambda(1405)$ to $\bar{K} N$ and $\pi \Sigma$ are important parameters to understand the non-mesonic transitions $\Lambda(1405) N \rightarrow Y N(Y=\Lambda$ or $\Sigma)$, which may be dominant processes of the nonmesonic $\bar{K}$ absorption in nuclei.

\section{Conclusion}

The $\Lambda(1405)$ is the "gift" of the meson-baryon dynamics. Due to the strong attractions in the $\bar{K} N$ and $\pi \Sigma$ channels, there exists two resonance poles around the $\Lambda(1405)$ energies. One 
is essentially a $\bar{K} N$ quasibound state just below the $\bar{K} N$ threshold, the other is a $\pi \Sigma$ resonance having a wide width and strong couping to the $\pi \Sigma$ state. The interference of these two states forms a single resonance peak in the $\pi \Sigma$ invariant mass spectra. Therefore the $\pi \Sigma$ invariant mass spectra depend on the initial channel for the $\Lambda(1405)$ production. The $\Lambda(1405)$ produced by the $\bar{K} N$ channel can be observed by $K^{-} d \rightarrow \Lambda(1405) n$. For better understanding of the $\Lambda(1405)$ structure and $\bar{K}$ nuclear few-body systems, any experimental information of the $\pi \Sigma \rightarrow \pi \Sigma$ scattering are desired, especially the $\pi \Sigma$ scattering length can fix the lower pole position of $\Lambda(1405) . K \bar{K} N$ can be another example of the $\bar{K}$ nuclear few-body systems. It is found that the $K \bar{K} N$ quasi-bound system is interpreted as coexistence state of $\Lambda(1405)$ and $a_{0}(980)$ clusters and $\bar{K}$ is a constituent of both $\Lambda(1405)$ and $a_{0}(980)$ at the same time.

\section{Acknowledgments}

This work is supported in part by the Grant for Scientific Research (No. 20028004) from Japan Society for from the Ministry of Education, Culture, Sports, Science and Technology (MEXT) of Japan. A part of this work is done under Yukawa International Project for QuarkHadron Sciences (YIPQS).

\section{References}

[1] R. H. Dalitz and S. F. Tuan, Phys. Rev. Lett. 2, 425 (1959); Annals Phys. 10, 307 (1960).

[2] N. Kaiser, P. B. Siegel, and W. Weise, Nucl. Phys. A594, 325 (1995).

[3] E. Oset and A. Ramos, Nucl. Phys. A635, 99 (1998).

[4] J. A. Oller and U. G. Meissner, Phys. Lett. B500, 263 (2001).

[5] E. Oset, A. Ramos, and C. Bennhold, Phys. Lett. B527, 99 (2002).

[6] T. Hyodo, S. I. Nam, D. Jido, and A. Hosaka, Phys. Rev. C 68, 018201 (2003); Prog. Theor. Phys. 112, 73 (2004).

[7] D. Jido, J. A. Oller, E. Oset, A. Ramos and U. G. Meissner, Nucl. Phys. A 725 (2003) 181.

[8] B. Borasoy, R. Nissler and W. Weise, Eur. Phys. J. A25 (2005) 79.

[9] B. Borasoy, U.G. Meissner, and R. Nissler, Phys. Rev. C 74, 055201 (2006).

[10] R. J. Hemingway, Nucl. Phys. B 253, 742 (1985).

[11] T. Hyodo and W. Weise, Phys. Rev. C77 (2008) 035204.

[12] D. Jido, E. Oset and T. Sekihara, Eur. Phys. J. A 42 (2009) 257.

[13] O. Braun et al., Nucl. Phys. B 129, 1 (1977).

[14] J. Yamagata-Sekihara, T. Sekihara, D. Jido, in preparation.

[15] D. Jido, E. Oset and A. Ramos, Phys. Rev. C 66 (2002) 055203.

[16] Y. Ikeda and T. Sato, Phys. Rev. C 76 (2007) 035203.

[17] Y. Ikeda, T. Hyodo, D. Jido, H. Kamano, T. Sato, K. Yazaki, in preparation.

[18] Y. Akaishi and T. Yamazaki, Phys. Rev. C 65, 044005 (2002).

[19] N. V. Shevchenko, A. Gal, and J. Mares, Phys. Rev. Lett. 98, 082301 (2007); N. V. Shevchenko, A. Gal, J. Mares and J. Revai, Phys. Rev. C 76, 044004 (2007).

[20] A. Dote, T. Hyodo and W. Weise, Phys. Rev. C79, 014003 (2009).

[21] D. Jido and Y. Kanada-En'yo, Phys. Rev. C 78 (2008) 035203; Y. Kanada-En'yo and D. Jido, Phys. Rev. C 78 (2008) 025212.

[22] Y. Ikeda and T. Sato, Phys. Rev. C 79 (2009) 035201.

[23] T. Hyodo, talk in this conference; T. Hyodo, D. Jido and A. Hosaka, Phys. Rev. C 78 (2008) 025203.

[24] T. Hyodo, D. Jido and L. Roca, Phys. Rev. D 77 (2008) 056010; L. Roca, T. Hyodo and D. Jido, Nucl. Phys. A 809 (2008) 65.

[25] T. Sekihara, T. Hyodo and D. Jido, Phys. Lett. B 669 (2008) 133.

[26] A. Martinez Torres, K. P. Khemchandani and E. Oset, Phys. Rev. C 77, 042203 (2008).

[27] T. Yamazaki and Y. Akaishi, Phys. Rev. C 76, 045201 (2007).

[28] T. Sekihara, talk in this conference; T. Sekihara, D. Jido and Y. Kanada-En'yo, Phys. Rev. C 79 (2009) 062201. 